\title{
A NEW CURVE ALGEBRAICALLY BUT NOT RATIONALLY UNIFORMIZED BY RADICALS*
}

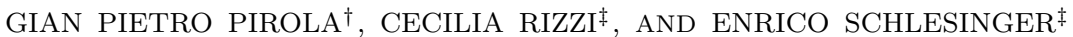

\begin{abstract}
We give a new example of a curve $C$ algebraically, but not rationally, uniformized by radicals. This means that $C$ has no map onto $\mathbb{P}^{1}$ with solvable Galois group, while there exists a curve $C^{\prime}$ that maps onto $C$ and has a finite morphism to $\mathbb{P}^{1}$ with solvable Galois group. We construct such a curve $C$ of genus 9 in the second symmetric product of a general curve of genus 2 . It is also an example of a genus 9 curve that does not satisfy condition $S(4,2,9)$ of Abramovich and Harris.
\end{abstract}

Key words. Monodromy groups, Galois groups, projective curves.

AMS subject classifications. $14 \mathrm{H} 10,14 \mathrm{H} 30,20 \mathrm{~B} 25$.

Introduction. Every smooth projective complex curve $C$ arises as a branched covering of the projective line $\mathbb{P}^{1}$, and its function field is a finite extension of the rational field $\mathbb{C}(x)$. However, it is a difficult problem to find a method for classifying all possible covering maps $C \rightarrow \mathbb{P}^{1}$. As explained by M. Fried in [8], Zariski tackled this problem for the general curve of genus $g$ (here and in the rest of this paper general means outside a countable union of closed subvarieties). In particular, in [21] Zariski proves what he regarded as the analogue for curves of Abel's theorem on the nonsolvability by radicals of a general algebraic equation of degree $\geq 5$ : the general curve $C$ of genus $g>6$ is not a covering of $\mathbb{P}^{1}$ with solvable Galois group. Zariski then goes on and speculates that it would still be possible, though unlikely, that there exist a finite covering $C^{\prime} \rightarrow C$ with $C^{\prime}$ representable as a solvable covering of $\mathbb{P}^{1}$. This problem has no analogue in the Galois theory of algebraic equations, because of the many different ways a curve can be viewed as a covering of $\mathbb{P}^{1}$.

To fix the terminology, the Galois group of a branched covering $C \rightarrow C_{0}$ of smooth curves is the Galois group of the Galois closure of the finite field extension $\mathbb{C}(C) / \mathbb{C}\left(C_{0}\right)$; it coincides with the monodromy group of the unramified covering obtained removing the branch divisor from $C_{0}$. We say the covering is solvable if its Galois group is solvable $[13,17]$. A curve $C$ is rationally uniformized by radicals if there exists a solvable covering map $C \rightarrow \mathbb{P}^{1}$, and is algebraically uniformized by radicals is there exists a finite covering $C^{\prime} \rightarrow C$ with $C^{\prime}$ rationally uniformized by radicals. Zariski's theorem then says that the general curve of genus $g>6$ is not rationally uniformized by radicals, and his question is whether $C$ might still be algebraically uniformized by radicals. It is not obvious that the two notions are really distinct: the first example of a curve that is algebraically, but not rationally, uniformized by radicals was given in [18] building on work of Debarre and Fahlaoui [6]. In this paper we give a new example. We feel that it is of utmost importance to investigate new examples to enhance our understanding of the difficult problem of describing the possible representations of the general curve $C$ of genus $g$ as a covering of $\mathbb{P}^{1}$. Let us recall that in recent years there has been a lot of research concerning Galois groups

*Received July 9 , 2010; accepted for publication October 19, 2012. Partially supported by: 1) MIUR PRIN 2007: Moduli, strutture geometriche e loro applicazioni; 2) Gnsaga. The second named author was also partially supported by a post-doc scholarship of Politecnico di Milano.

${ }^{\dagger}$ Department of Mathematics, University of Pavia, Via Ferrata 1, 27100 Pavia, Italy (gianpietro. pirola@unipv.it).

$\ddagger$ Dipartimento di Matematica "F. Brioschi”, Politecnico di Milano, Piazza Leonardo da Vinci 32, 20133 Milano, Italy (\{cecilia.rizzi; enrico.schlesinger\}@polimi.it). 
of coverings $C \rightarrow B$ when the genus of $C$ is fixed: see for example [12] and its list of references. In particular, it is now known that in the moduli space of genus $g$ curves the locus of curves that are rationally uniformized by radicals is contained in a proper Zariski closed subset [16, Theorem 1.6] and [11, Theorem 2.4].

Debarre and Fahlaoui were motivated by a related problem posed by Abramovich and Harris [1], who formulated the statement:

STATEMENT $S(d, h, g)$ : Suppose $C^{\prime} \rightarrow C$ is a nonconstant map of smooth curves with $C$ of genus $g$. If $C^{\prime}$ admits a map of degree $d$ or less to a curve of genus $h$ or less, then so does $C$.

Abramovich and Harris noted that $S(d, 0, g)$ is true for elementary reasons, proved $S(2,1, g), S(3,1, g)$ and $S(4,1, g)$ for $g \neq 7$, and showed that $S(3,2,5)$ is false. Thus they asked for which values of $d, h$ and $g$ the statement holds. In [6] it is shown that $S(4,1,7)$ is false, and in [18] we showed that the curve of genus 7 providing the counterexample is algebraically, but not rationally, uniformized by radicals. Debarre and Fahlaoui construct their example $C$ on the second symmetric product $\operatorname{Sym}^{2}(E)$ of an elliptic curve. It is clear by their construction that $C$ has a covering $C^{\prime}$ that admits a map of degree 4 or less to $E$; in particular, $C$ is algebraically uniformized by radicals. The key step in disproving $S(4,1,7)$ in $[6]$ is to show that $C$ has no $4: 1$ map to $\mathbb{P}^{1}$, and from this we were able to deduce in [18] that $C$ is not rationally uniformized by radicals.

In this paper we show the existence of curves of genus 9 that are algebraically, but not rationally, uniformized by radicals, and that provide counterexamples to $S(4,2,9)$. We construct such curves in a linear system $|C|$ on the second symmetric product $X=\operatorname{Sym}^{2}(Y)$ of a curve of genus 2. The hard part of the proof again consists in showing the general curve in $|C|$ has no $g_{4}^{1}$. The technique we use is different from that of [6] and comes from an idea of Tyurin [20]. Roughly, the idea is to consider in the Hilbert scheme $\mathrm{Hilb}^{4}(X)$ the set $\mathcal{S}$ of all divisors appearing in a $g_{4}^{1}$ of a curve in the family $|C|$, and to bound the dimension of this set in order to show the general curve in $|C|$ cannot contain such a divisor. To find such a bound, one blows down the canonical divisor of $X=S y m^{2}(Y)$ to obtain the Jacobian surface $S$ of $Y$, and then notes that the fibers of the Abel sum map $\operatorname{Hilb}^{4}(S) \rightarrow \operatorname{Alb}(S)=S$ are symplectic varieties [3]. The set $\mathcal{S}$ is contained in such a fiber and, being rationally connected, must lie in a Lagrangian subvariety. This provides the desired bound for the dimension of $\mathcal{S}$.

The paper is structured as follows. In Section 1 we introduce the notation and terminology, and state some well known results we will use in the paper. In Section 2 we construct the family of curves that will eventually provide the example we are looking for, and show it has dimension ten. In Section 3, which is the technical heart of the paper, we show that a general curve $C$ in our family has no $g_{4}^{1}$. In Section 4 we show by a dimension count that a general curve $C$ in a ten dimensional family of genus nine curves cannot be a covering of $\mathbb{P}^{1}$ of degree $d \geq 5$ with a primitive and solvable Galois group. Putting together the results of these two sections in Section 5 we conclude that $C$ is not rationally uniformized by radicals. On the other hand, by construction every curve in the family is algebraically uniformized by radicals, and we therefore obtain examples of genus 9 curves that are algebraically, but not rationally uniformized by radicals.

1. Notation and preliminary results. In this section we collect some well known facts that we will use in the sequel of the paper. We begin recalling the 
cohomology of divisors of small degree on a curve of genus 2 .

Proposition 1.1. Let $Y$ be a smooth irreducible projective complex curve of genus 2 , and let $B$ be a divisor on $Y$. Denote by $p \mapsto p^{\prime}$ the hyperelliptic involution on $Y$, so that $q=p^{\prime}$ if and only if $p+q$ is a canonical divisor $K_{Y}$ on $Y$. Then

i) if $\operatorname{deg}(B)=2$, then $h^{0}\left(Y, \mathcal{O}_{Y}(B)\right)=2$ if $B$ is a canonical divisor, $h^{0}\left(Y, \mathcal{O}_{Y}(B)\right)=1$ otherwise.

ii) if $\operatorname{deg}(B)=3$, then $B$ is nonspecial, $h^{0}\left(Y, \mathcal{O}_{Y}(B)\right)=2$, and

a) either $B \sim K_{Y}+p$, in which case $p$ is a base point of $|B|$;

b) or $B-K_{Y}$ is not effective, in which case $|B|$ has no base points, and $\phi_{B}: Y \rightarrow \mathbb{P}^{1}$ is a morphism of degree 3 .

iii) if $\operatorname{deg}(B)=4$, then $B$ is nonspecial, the linear series $|B|$ is base point free and defines a morphism $\phi_{B}: Y \rightarrow \mathbb{P}^{2}$. Furthermore:

a) either $B \sim 2 K_{Y}$, in which case $\phi_{B}$ is the hyperelliptic involution $Y \rightarrow \mathbb{P}^{1}$ followed by the 2-uple embedding $\mathbb{P}^{1} \rightarrow \mathbb{P}^{2}$; in particular, every effective divisor in $\left|2 K_{Y}\right|$ is the sum $p+p^{\prime}+q+q^{\prime}$ of two canonical divisors;

b) or $B \sim K_{Y}+p+q$ where $q \neq p^{\prime}$, in which case $\phi_{B}: Y \rightarrow \mathbb{P}^{2}$ is birational onto a plane quartic curve, which has a node if $p \neq q$ or a cusp if $p=q$; $\phi_{B}$ separates any pair of points of $Y$ except for the pair $(p, q)$.

We will construct our example on the second symmetric product $X=\operatorname{Sym}^{2}(Y)$ of a curve of genus two $Y$. The surface $X$ has irregularity $q(X)=g(Y)=2$ and geometric genus $p_{g}(X)=\left(\begin{array}{c}g(Y) \\ 2\end{array}\right)=1$, hence $\chi\left(\mathcal{O}_{X}\right)=0$.

We identify points of $X$ with degree 2 effective divisors $p+q$ on $Y$. The quotient map

$$
\pi: Y \times Y \longrightarrow X, \quad \pi(p, q)=p+q
$$

exhibits $X$ as the quotient of $Y \times Y$ by the involution $\sigma(p, q)=(q, p)$. Let $\tilde{\Delta}$ denote the diagonal in $Y \times Y$, and let $\Delta=\pi(\tilde{\Delta})=\{2 p: p \in Y\}$. The map $\pi$ is a double cover ramified along $\Delta$, so that $\pi_{*} \mathcal{O}_{Y \times Y}=\mathcal{O}_{X} \oplus \mathcal{L}^{-1}$ where $\mathcal{L}$ is a line bundle satisfying $\mathcal{L}^{\otimes 2}=\mathcal{O}_{X}(\Delta)$. In particular $\frac{\Delta}{2}$ is an integral divisor class on $X$, and $\pi^{*}\left(\frac{\Delta}{2}\right)=\tilde{\Delta}$.

Given a point $p \in Y$, we denote by $H_{p}$ the curve

$$
H_{p}=\{p+q \in X \mid q \in Y\} \subset X .
$$

One knows that the canonical divisors of $Y$ and $X$ are related as follows: if $p+p^{\prime}$ is a canonical divisor on $Y$, then the canonical divisor of $X$ is $K_{X} \sim H_{p}+H_{p^{\prime}}-\frac{\Delta}{2}$.

Another way to look at $X$ is via the natural map $X=\operatorname{Div}^{(2)}(Y) \rightarrow \operatorname{Pic}^{(2)}(Y)$, which exhibits $X$ as the blow up of the Jacobian variety $\operatorname{Pic}^{(2)}(Y)$ at the point corresponding to the canonical divisor $K_{Y}$. The exceptional divisor $E \cong \mathbb{P}^{1}$ is therefore the unique effective canonical divisor on $X$. In particular, $E \sim H_{p}+H_{p^{\prime}}-\frac{\Delta}{2}$.

Any divisor $B=\sum_{p} n_{p} p$ on $Y$ gives rise to a divisor $\alpha(B)=\sum_{p} n_{p} H_{p}$ on $X$. The map $\operatorname{Pic}(Y) \rightarrow \operatorname{Pic}(X)$ induced by $\alpha$ is injective, and in fact $\operatorname{Pic}(X)$ contains a subgroup isomorphic to $\alpha(\operatorname{Pic}(Y)) \oplus \mathbb{Z}[E]$. Furthermore, a divisor on $X$ is numerically equivalent to zero if and only if it is linearly equivalent to a divisor of the form $\alpha(B)=\sum_{p} n_{p} H_{p}$ with $\sum n_{p}=0$.

When $Y$ has general moduli, the endomorphism ring of the Jacobian variety $\operatorname{Jac}(Y)$ is $\mathbb{Z}$ generated by the identity [14], and from this it follows $\operatorname{Pic}(X)=$ $\alpha(\operatorname{Pic}(Y)) \oplus \mathbb{Z}[E]$. 
One can easily compute the cohomology of the divisors $\mathcal{O}_{X}(\alpha(B))$ :

Proposition 1.2. Let $B=\sum_{p} n_{p} p$ be a divisor on $Y$, and let $\alpha(B)=\sum_{p} n_{p} H_{p}$ be the corresponding divisor on $X$. Then $\alpha(B)$ is effective if and only if $B$ is effective. Furthermore

i) If $\operatorname{deg}(B)=1$ and $B=p$ is effective, then

$$
h^{0}\left(X, \mathcal{O}_{X}\left(H_{p}\right)\right)=h^{1}\left(X, \mathcal{O}_{X}\left(H_{p}\right)\right)=1, \quad h^{2}\left(X, \mathcal{O}_{X}\left(H_{p}\right)\right)=0
$$

ii) if $\operatorname{deg}(B)=2$ and $B \sim K_{Y}$, then

$$
h^{0}\left(X, \mathcal{O}_{X}(\alpha(B))\right)=3, \quad h^{1}\left(X, \mathcal{O}_{X}(\alpha(B))\right)=2, \quad h^{2}\left(X, \mathcal{O}_{X}(\alpha(B))\right)=0
$$

iii) if $\operatorname{deg}(B)=2$ and $B \nsim K_{Y}$, then

$$
h^{0}\left(X, \mathcal{O}_{X}(\alpha(B))\right)=1, \quad h^{1}\left(X, \mathcal{O}_{X}(\alpha(B))\right)=h^{2}\left(X, \mathcal{O}_{X}(\alpha(B))\right)=0
$$

iv) if $\operatorname{deg}(B)=b \geq 3$, then

$$
h^{0}\left(X, \mathcal{O}_{X}(\alpha(B))\right)=\frac{1}{2} b(b-1), \quad h^{1}\left(X, \mathcal{O}_{X}(\alpha(B))\right)=h^{2}\left(X, \mathcal{O}_{X}(\alpha(B))\right)=0
$$

Let $N^{1}(X)$ denote the Néron-Severi group of $X$ (divisors modulo numerical equivalence for which we use the symbol $\equiv)$. We denote by $H$ the class of $H_{p}$ in $N^{1}(X)$, while we keep using the symbols $\Delta$ and $E$ for the classes of $\Delta$ and $E$ in $N^{1}(X)$. From the above description of $\operatorname{Pic}(X)$ we see that $N^{1}(X)$ contains the subgroup

$$
\mathbb{Z}[H] \oplus \mathbb{Z}[E]
$$

and $N^{1}(X)=\mathbb{Z}[H] \oplus \mathbb{Z}[E]$ when $Y$ has general moduli. Note that

$$
H^{2}=1 \quad H \cdot E=1 \quad E^{2}=-1 .
$$

Proposition 1.3. In the Néron-Severi group of $X$

- some positive multiple of an integral class $a H-b E$ is effective if and only if $a \geq 0$ and $a \geq 2 b$.

- an integral class $a H-b E$ is ample (respectively nef) if and only if $a>-b$ and $a>3 b$ (respectively $a \geq-b$ and $a \geq 3 b$ ).

Proof. The first statement follows from the fact that $H$ is ample and the two effective curves $\Delta \equiv 4 H-2 E$ and $E$ have negative self-intersection.

To check the second statement, let $R_{1} \equiv H+E$ and $R_{2} \equiv 3 H-E \equiv H+\frac{\Delta}{2}$. Then $R_{1} \cdot E=R_{2} \cdot \Delta=0$. Since $H$ is ample, it follows that $R_{1}$ and $R_{2}$ are nef but not ample.

2. A ten dimensional family of genus nine curves. In this section we construct a 10 dimensional family of curves of genus 9 whose general member we will eventually show to be algebraically but not rationally uniformized by radicals. We keep the notation we introduced in the previous section for divisors on the surface $X=\operatorname{Sym}^{2}(Y)$, where $Y$ denotes a smooth projective curve of genus 2 . We begin by showing the existence of smooth genus 9 curves numerically equivalent to $3 H+E$ on $X$. 
Proposition 2.1. Let $L$ be a divisor on $X$ numerically equivalent to $3 H+E$. Then

$$
h^{0}\left(\mathcal{O}_{X}(L)\right)=6, \quad h^{1}\left(\mathcal{O}_{X}(L)\right)=h^{2}\left(\mathcal{O}_{X}(L)\right)=0 .
$$

Furthermore:

- the linear system $|L|$ is base point free, and defines a morphism $\phi_{L}: X \rightarrow \mathbb{P}^{5}$ that maps $X$ birationally onto its image.

- the general curve $C \in|L|$ is smooth and irreducible; the genus of such a curve $C$ is $g(C)=9$ and its self-intersection is $C^{2}=14$.

Proof. Any divisor numerically equivalent to $3 H$ is ample, hence $h^{1}\left(\mathcal{O}_{X}(L)\right)=$ $h^{2}\left(\mathcal{O}_{X}(L)\right)=0$ by Kodaira vanishing theorem. Therefore

$$
h^{0}\left(\mathcal{O}_{X}(L)\right)=\chi\left(\mathcal{O}_{X}(L)\right)=\frac{1}{2}\left(L-K_{X}\right) \cdot L+\chi \mathcal{O}_{X}=6 .
$$

Since $L \equiv K_{X}+3 H$ and $H$ is ample, it follows from Reider's Theorem [19] that $|L|$ is base point free, hence the generic curve $C \in|L|$ is smooth (the ground field is $\mathbb{C}$ ). Also $L$ itself is ample, hence any curve in $|L|$ is (numerically) connected (cf. [9] Ex. 13 p. 24).

We need to check that $\phi_{L}$ is birational onto its image. The divisor $L$ is linearly equivalent to $E+\alpha\left(B_{0}\right)$ where $B_{0}$ is a divisor of degree 3 on $Y$, thus $\mathcal{O}_{X}(L) \cong$ $\mathcal{O}_{X}\left(E+H_{p}+H_{q}+H_{r}\right)$ where $(p, q, r)$ are three points of $Y$.

By [19], Remark 1.2.2, if two points of $X$ are not separated by $|L|$, then there is a curve $F$ numerically equivalent to $H$ passing through the two points. By Proposition $1.2 F=H_{x}$ for some $x \in Y$. We will now show that for every $x$ the linear system $|L|$ separates all but one pair of points of $H_{x}$, unless $\mathcal{O}_{Y}(p+q+r) \cong \mathcal{O}_{Y}\left(x+K_{Y}\right)$ so that $x$ is the unique base point of $\mathcal{O}_{Y}(p+q+r)$. This shows that $\phi_{L}$ is one to one on $X$ except on an at most one dimensional locus, and concludes the proof.

From the exact sequence

$$
0 \rightarrow \mathcal{O}_{X}\left(L-H_{x}\right) \rightarrow \mathcal{O}_{X}(L) \rightarrow \mathcal{O}_{H_{x}}(L) \rightarrow 0
$$

we see every section of $\mathcal{O}_{H_{x}}(L)$ arises from a section of $\mathcal{O}_{X}(L)$ because $H^{1}\left(\mathcal{O}_{X}(L-\right.$ $\left.\left.H_{x}\right)\right)=0$ by Kodaira vanishing. Thus it is enough to show that $\mathcal{O}_{H_{x}}(L)$ separates all but a pair of points of $H_{x}$ if $x$ is not a base point of $\mathcal{O}_{Y}(p+q+r)$.

Thus we assume $x$ is not a base point of $\mathcal{O}_{Y}(p+q+r)$, and we can then take $p, q$ and $r$ distinct from $x$. The restriction $\mathcal{O}_{H_{x}}(L)$ of $L$ to the curve $H_{x} \cong Y$ is $\mathcal{O}_{Y}\left(x^{\prime}+p+q+r\right)$, where $x^{\prime}$ is the conjugate point of $x$ so that $x+x^{\prime} \sim K_{Y}$. If we had $x^{\prime}+p+q+r \sim 2 K_{Y}$, then $p+q+r \sim x+K_{Y}$ contradicting the assumption that $x$ is not a base point of $\mathcal{O}_{Y}(p+q+r)$. Thus $x^{\prime}+p+q+r \sim 2 K_{Y}$, and so $\mathcal{O}_{H_{x}}(L)$ separates all but one pair of points of $H_{x}$ by Proposition 1.1.

We outline now the standard arguments that allow one to compute the dimension of the family of genus 9 curves $C$ arising as in the previous proposition.

Proposition 2.2. In the moduli space of curve of genus 9 there is a 10 dimensional family of curves whose general member is a curve $C$ numerically equivalent to $3 H+E$ on a surface $X=\operatorname{Sym}^{2}(Y)$, where $Y$ is a general curve of genus 2 .

Proof. Let $f: \mathcal{Y} \rightarrow B$ be a smooth family of genus two curves such that the associated moduli map $B \rightarrow \mathcal{M}_{2}$ is generically finite and dominant, and let

$$
p: \mathcal{X}=\mathcal{Y} \times_{B} \mathcal{Y} / \mathcal{S}_{2} \rightarrow B
$$


be the corresponding family of symmetric products.

Consider the relative Hilbert scheme $\mathcal{H}=\operatorname{Hilb}(\mathcal{X} / B)$. We fix a closed point $b \in B$, and let $X=\mathcal{X}_{b}$ and $Y=\mathcal{Y}_{b}$. Then the fiber $\mathcal{H}_{b}$ is the Hilbert scheme $\operatorname{Hilb}(X)$.

Fix a smooth curve $C \equiv 3 H+E$ on $X$. Since $H^{1}\left(\mathcal{O}_{X}(C)\right)=0$, the Hilbert schemes $\mathcal{H}$ and $\mathcal{H}_{b}$ are smooth at the point $[C]$ corresponding to $C$, and there exists an exact sequence of tangent spaces

$$
0 \rightarrow T_{[C]} \mathcal{H}_{b} \rightarrow T_{[C]} \mathcal{H} \rightarrow T_{b} B \rightarrow 0 .
$$

Claim. If $\alpha: \mathcal{H}_{b} \rightarrow \mathcal{M}_{9}$ denotes the moduli map, its differential

$$
d \alpha: T_{[C]} \mathcal{H}_{b} \cong H^{0}\left(C, \mathcal{O}_{C}(C)\right) \rightarrow T_{[C]} \mathcal{M}_{b} \cong H^{1}\left(C, \mathcal{T}_{C}\right)
$$

is injective

Since the kernel of $d \alpha$ is (a quotient of) $H^{0}\left(\left.\mathcal{T}_{X}\right|_{C}\right.$ ), it is enough to show the latter group vanishes. For this, we look at the blow up map $\rho: X \rightarrow S=\operatorname{Pic}^{2}(Y) \cong \operatorname{Jac}(Y)$. Restricting the exact sequence

$$
0 \rightarrow \mathcal{T}_{X} \rightarrow \rho^{*} \mathcal{T}_{S} \cong \mathcal{O}_{X} \oplus \mathcal{O}_{X} \rightarrow \mathcal{N}_{\rho} \cong \mathcal{O}_{E}(-E) \cong \mathcal{O}_{\mathbb{P}^{1}}(1) \rightarrow 0
$$

to the curve $C$ one obtains a new exact sequence

$$
\left.\left.0 \rightarrow \mathcal{T}_{X}\right|_{C} \rightarrow\left(\rho^{*} \mathcal{T}_{S}\right)\right|_{C} \cong \mathcal{O}_{C}^{\oplus 2} \rightarrow \mathcal{O}_{Z} \rightarrow 0
$$

where $Z$ is the length 2 zero dimensional intersection of $C$ and $E$. One checks

$$
H^{0}\left(\left.\left(\rho^{*} \mathcal{T}_{S}\right)\right|_{C}\right) \rightarrow H^{0}\left(\mathcal{O}_{Z}\right)
$$

is an isomorphism, hence its kernel $H^{0}\left(\left.\mathcal{T}_{X}\right|_{C}\right)$ vanishes, proving the claim.

Now let $\beta: \mathcal{H} \rightarrow \mathcal{M}_{b}$ denote the moduli map on the relative Hilbert scheme. Then $d \beta$ induces a map $T_{b} B \cong H^{1}\left(\mathcal{T}_{Y}\right) \rightarrow \operatorname{Coker}(d \alpha)$. Now observe that

$$
\operatorname{Coker}(d \alpha) \cong H^{1}\left(\mathcal{T}_{C}\right) / \operatorname{Im}\left(H^{0}\left(\mathcal{O}_{C}(C)\right) \hookrightarrow H^{1}\left(\left.\left(\mathcal{T}_{X}\right)\right|_{C}\right)\right.
$$

Thus we obtain a map

$$
\phi: T_{b} B \cong H^{1}\left(\mathcal{T}_{Y}\right) \rightarrow H^{1}\left(\left.\left(\mathcal{T}_{X}\right)\right|_{C}\right) .
$$

Assume for the moment that $\phi$ is injective. Then $d \beta: T_{[C]} \mathcal{H} \rightarrow T_{[C]} \mathcal{M}_{b}$ is injective, and this proves the proposition because

$$
h^{0}\left(\mathcal{O}_{C}(C)\right)+h^{1}\left(\mathcal{T}_{Y}\right)=7+3=10 .
$$

To show $\phi$ is injective, notice that it factors through the map $\psi: H^{1}\left(\mathcal{T}_{Y}\right) \rightarrow$ $H^{1}\left(\mathcal{T}_{X}\right)$ that associates to a in infinitesimal deformation of $Y$ the corresponding deformation of $X$. The map $\psi$ is injective: identifying $Y$ with the diagonal $\Delta \subset X$, we see the kernel of $\psi$ is contained in the kernel of $H^{1}\left(\mathcal{T}_{\Delta}\right) \rightarrow H^{1}\left(\left.\left(\mathcal{T}_{X}\right)\right|_{\Delta}\right)$, hence in $H^{0}\left(\mathcal{O}_{\Delta}(\Delta)\right)$. The latter group vanishes, hence $\psi$ is injective.

Finally, $\phi$ is obtained composing $\psi$ with

$$
H^{1}\left(\mathcal{T}_{X}\right) \rightarrow H^{1}\left(\left.\left(\mathcal{T}_{X}\right)\right|_{C}\right)
$$

whose kernel is $H^{1}\left(\mathcal{T}_{X}(-C)\right)$. This cohomology group is contained in $H^{1}\left(\mathcal{O}_{X}(-C)^{\oplus 2}\right)$, which vanishes because $\mathcal{O}_{X}(C)$ is ample. Thus $\phi: H^{1}\left(\mathcal{T}_{Y}\right) \rightarrow$ $H^{1}\left(\left.\left(\mathcal{T}_{X}\right)\right|_{C}\right)$ is injective, and this concludes the proof. 
3. The general curve in the family has no $g_{4}^{1}$. This section contains the main technical difficulty of the paper, which is to prove that the general curve in our family of genus 9 curves has no $g_{4}^{1}$. To be more precise, recall that the Jacobian of a general smooth projective curve is simple [14]. Therefore, if $Y$ is a general curve of genus 2, the Néron Severi group of $X=\operatorname{Sym}^{2}(Y)$ is generated by the classes of $H$ and $E$. If this is the case and $L$ is a divisor on $X$ numerically equivalent to $3 H+E$, we will show that in the linear system $|L|$ there is an open dense subset of smooth curves that have no $g_{4}^{1}$. Before we can prove this, we need to establish the fact that these curves have no map onto a non rational curve.

Proposition 3.1. Suppose $Y$ is a smooth projective curve of genus 2 whose Jacobian is simple, and let $X=\operatorname{Sym}^{2}(Y)$. Suppose $L$ is a divisor on $X$ numerically equivalent to $3 H+E$, and $C$ is a general curve in the linear system $|L|$. If $D$ is a smooth curve for which there is a finite morphism $C \rightarrow D$ of degree $d \geq 2$, then $D$ is rational.

Proof. Suppose $f: C \rightarrow D$ is a finite morphism of smooth curves of degree $d \geq 2$. By [4, Theorem 1.1] the Jacobian of $C$ satisfies

$$
\operatorname{End}(\operatorname{Jac}(C))=\mathbb{Z} \times \operatorname{End}(\operatorname{Alb}(X))=\mathbb{Z} \times \operatorname{End}(\operatorname{Jac}(Y))
$$

Since $\operatorname{End}(\operatorname{Jac}(Y))=\mathbb{Z}$, the abelian subvarieties of $\operatorname{Jac}(C)$ have dimension $0,2,7$ or 9. It follows that, if $D$ is not rational and $d \geq 2$, then $g(D)=2$ and there is an isogeny $\phi: \operatorname{Jac}(Y) \rightarrow \operatorname{Jac}(D)$ which factors through the map $\operatorname{Jac}(C) \rightarrow \operatorname{Jac}(D)$ induced by $f$.

Let $C_{0}$ be the inverse image of $C$ in $J a c(Y)$ under the map $J a c(Y) \rightarrow J a c(C)$. Since $\phi: \operatorname{Jac}(Y) \rightarrow \operatorname{Jac}(D)$ is étale, so is its restriction $\psi: C_{0} \rightarrow D$. But $\psi$ factors through $f: C \rightarrow D$, thus $f$ is étale.

The family of genus 9 curves that are étale covers of a genus two curve has dimension $3=\operatorname{dim} \mathcal{M}_{2}$. On the other hand, $C$ varies in $|L| \cong \mathbb{P}^{5}$, and $|L|$ that by the proof of (2.2) maps with zero dimensional fibers to the moduli space $\mathcal{M}_{9}$. Therefore the general $C \in|L|$ is not an étale cover of a genus two curve, finishing the proof of the lemma. $\mathrm{C}$

THEOREM 3.2. Suppose $Y$ is a smooth projective curve of genus 2 whose Jacobian is simple, and let $X=\operatorname{Sym}^{2}(Y)$. Suppose $L$ is a divisor on $X$ numerically equivalent to $3 H+E$, and $C$ is a general curve in the linear system $|L|$. Then $C$ has no $g_{4}^{1}$.

Proof. Suppose by way of contradiction that the general curve in $|L|$ has gonality $d \leq 4$, and therefore has a base point free $g_{d}^{1}$ with $2 \leq d \leq 4$. We distinguish two cases, according to whether the $g_{d}^{1}$ is unique or not.

Case 1. Assume first the general curve $C$ in the family has a unique $g_{d}^{1}$.

The natural map $X=\operatorname{Sym}^{2}(Y) \rightarrow \operatorname{Pic}^{2}(Y)$ identifies $X$ with the blow up of the abelian surface $S=J a c(Y)$ at the origin, and $E$ is the exceptional divisor. By a theorem of Fogarty's [7] the Hilbert scheme $\mathcal{H}=H_{i l b}(S)$ parametrizing zero dimensional subschemes of $S$ of length $d$ is a smooth and irreducible projective variety of dimension $2 d$. We will identify a zero dimensional subscheme $Z$ of $S$ that does not contain the origin $0_{S}$, with its preimage in $X$. Then it makes sense to look at the incidence variety

$$
\left\{(C, Z) \in|L| \times \mathcal{H}: C \text { is smooth, }, 0_{S} \notin Z, Z \subseteq C, h^{0}\left(C, \mathcal{O}_{C}(Z)\right) \geq 2\right\} .
$$


Let $W$ be an irreducible component of this locus that maps dominantly to $|L|$, and let $\pi_{1}$ and $\pi_{2}$ be the two projections of $W$ on $|L|$ and $\mathcal{H}$ respectively. Since the general curve in $|L|$ has a unique $g_{d}^{1}$, the map $\pi_{1}$ is dominant and its general fiber is a rational curve. Therefore $W$ is a rationally connected variety of dimension $6[10,5]$.

Now look at the Abel sum map

$$
\alpha: \mathcal{H}=\operatorname{Hilb}^{d}(S) \rightarrow \operatorname{Alb}(S)=S .
$$

Since $W$ is rationally connected, the image $\pi_{2}(W)$ of $W$ in $\mathcal{H}$ must be contained in a fiber $K$ of $\alpha$. The fiber $K$ is a symplectic variety of dimension 2(d-1)- see [3] . The pull back of the symplectic form to $W$ vanishes because $W$ is rationally connected, hence $\pi_{2}(W)$ is a Lagrangian subvariety of $K$. Therefore

$$
\operatorname{dim} \pi_{2}(W) \leq \frac{1}{2} \operatorname{dim} K=d-1
$$

and the generic fiber of $\pi_{2}$ has dimension at least $7-d$.

By Proposition 2.1 the linear system $|L|$ defines a morphism $\phi: X \rightarrow \mathbb{P}^{5}$ that maps $X$ birationally onto its image. Note that $\mathbb{P}^{5}$ is the dual projective space of $|L| \cong$ $\mathbb{P}\left(H^{0}(X, L)\right)$. For a closed subscheme $V \subset X$, we let $|L(-V)| \cong \mathbb{P}\left(H^{0}\left(X, L \otimes \mathcal{I}_{V}\right)\right)$ denote the linear system of curves in $|L|$ that contain $V$. Then the linear span of $\phi(V)$ in $\mathbb{P}^{5}$ is the subspace dual to $|L(-V)|$; the dimension of the linear span of $\phi(V)$ is therefore $4-\operatorname{dim}|L(-V)|$.

Now let $(C, Z)$ be a point of $W$. Then $|L(-Z)|$ contains the fiber $\pi_{2}^{-1}(Z)$, hence

$$
\operatorname{dim}|L(-Z)| \geq \operatorname{dim}\left(\pi_{2}^{-1}(Z)\right) \geq 7-d .
$$

It follows that the image $\phi(Z)$ of $Z$ in $\mathbb{P}^{5}$ is contained in a linear space of dimension $d-3$. Since $d \leq 4$, this says that $\phi(Z)$ is contained in a line. Since $\phi$ is birational, for $Z$ general the linear span of $\phi(Z)$ will be a line, hence $d=4$, and $\operatorname{dim}|L(-Z)|=3$. Since $\operatorname{dim}\left(\pi_{2}^{-1}(Z)\right) \geq 3$, we conclude that $\operatorname{dim}\left(\pi_{2}^{-1}(Z)\right)=3$ for a general $Z$ in $\pi_{2}(W)$, and therefore the general curve $C$ in $|L(-Z)|$ belongs to $\pi_{2}^{-1}(Z)$, that is, $h^{0}\left(\mathcal{O}_{C}(Z)\right) \geq 2$. By semicontinuity, $h^{0}\left(\mathcal{O}_{C}(Z)\right) \geq 2$ for every smooth $C$ in $|L(-Z)|$.

For a point $a \in X$ define

$$
\begin{aligned}
B_{a}^{0}= & \left\{x \in X: \text { there exists } Z \in \pi_{2}(W) \text { such that } a, x \in Z\right. \\
& \text { and } \left.h^{0} \mathcal{O}_{C}(Z) \geq 2 \text { for every smooth } C \text { in }|L(-Z)|\right\}
\end{aligned}
$$

and let $B_{a}$ denote the closure of $B_{a}^{0}$ in $X$. For a general choice of $a$, the dimension of $B_{a}$ is one. To see this, let $W_{a}$ denote the set of pairs $(C, Z)$ in $W$ for which $a \in Z$. Then $W_{a}$ has dimension 4 because $\pi_{1}$ maps $W_{a}$ generically onto $|L(-a)| \cong \mathbb{P}^{4}$, with zero dimensional fibers as there is a unique divisor in the $g_{4}^{1}$ of $C$ that contains $a$. Since the general fiber of $\pi_{2}: W_{a} \rightarrow \mathcal{H}$ has dimension 3 , the image of $W_{a}$ in the Hilbert scheme is a curve $T$. Therefore the restriction $U_{T}$ to $T$ of the universal family over $\mathcal{H}$ is also a curve, and so is $B_{a}$ which is the closure of the projection in $X$ of $U_{T}$ with the point $a$ removed.

We claim that, for a general $C_{0} \in|L(-a)|$,

$$
B_{a} \cdot C_{0}=m a+x_{1}+x_{2}+x_{3}
$$

where $Z_{0}=a+x_{1}+x_{2}+x_{3}$ is the unique element of the $g_{4}^{1}$ of $C_{0}$ that contains $a$, and $m \geq 0$. Indeed, since $C_{0}$ is general in $|L(-a)|$, it is smooth and it does not contain any 
of the finitely many points of $B_{a}-B_{a}^{0}$ except perhaps $a$. So, if $x \in B_{a} \cap C_{0} \backslash\{a\}$, there is $Z \in \pi_{2}(W)$ such that $a, x \in Z$ and $h^{0} \mathcal{O}_{C}(Z) \geq 2$ for every smooth $C \in|L(-Z)|$. Now

$$
3 \leq \operatorname{dim}|L(-Z)|=\operatorname{dim}|L-(a+x)|
$$

hence $|L(-Z)|=|L-(a+x)|$ and $C_{0} \in|L(-Z)|$. Then $h^{0} \mathcal{O}_{C_{0}}(Z) \geq 2$, and $Z=Z_{0}$ because there is a unique divisor in the $g_{4}^{1}$ of $C_{0}$ that contains $a$. In particular, $x \in\left\{x_{1}, x_{2}, x_{3}\right\}$ proving our claim.

The claim implies that the intersection of $\phi\left(B_{a}\right)$ with a general hyperplane of $\mathbb{P}^{5}$ through $\phi(a)$ is contained in a line: the hyperplane corresponds to $C_{0}$, and the line is the linear span of $\phi\left(Z_{0}\right)$. It follows that $\phi\left(B_{a}\right)$ is contained in a $\mathbb{P}^{2}$ through $\phi(a)$, hence

$$
\operatorname{dim}\left|L\left(-B_{a}\right)\right| \geq 2 .
$$

We conclude that $B_{a}$ and $C-B_{a}$ are effective, with $h^{0} \mathcal{O}_{X}\left(C-B_{a}\right) \geq 3$. Now we use the fact that the Néron Severi group of $X$ is generated by $E$ and $H$. Since $B_{a}$ moves with $a$, we see $B_{a} \neq E, 2 E$. On the other hand, $C-B_{a}$ can't be numerically equivalent to $H+2 E, H+E$ or $H$ because $h^{0} \mathcal{O}_{X}\left(C-B_{a}\right) \geq 3$. It then follows from Proposition 1.3 that $C-B_{a}$ is numerically equivalent to either $2 H$ or $2 H+E$, so that either $B_{a} \equiv H$ or $B_{a} \equiv H+E$.

Suppose $B_{a} \equiv H$. Then $B_{a}$ is one of the curves $H_{p}$ (with $p \in Y$ ). As $B_{a} . C=$ $H . C=4$, the $g_{4}^{1}$ on $C$ is $\left|H_{C}\right|$, where $H_{C}=H_{p} . C$. Now look at the exact sequence

$$
0 \rightarrow \mathcal{O}_{X}\left(H_{p}-C\right) \rightarrow \mathcal{O}_{X}\left(H_{p}\right) \rightarrow \mathcal{O}_{C}\left(H_{C}\right) \rightarrow 0 .
$$

Now $C-H \equiv 2 H+E=2 H+K_{X}$ is ample by 1.3 , hence $H^{1} \mathcal{O}_{X}\left(H_{p}-C\right)=0$, therefore $h^{0}\left(C, \mathcal{O}_{C}\left(H_{C}\right)\right)=1$, so $H_{C}$ cannot be a pencil, and this case does not occur.

Suppose now $B_{a} \equiv H+E$, that is, $B_{a}=H_{p}+E$ for some $p \in Y$. Then

$$
B_{a} \cdot C=(H+E) \cdot C=4+2=6
$$

contains the $g_{4}^{1}$, hence $h^{0}\left(\left(H_{p}+E\right)_{C}\right) \geq 2$. But from the exact sequence

$$
0 \rightarrow \mathcal{O}_{X}\left(H_{p}+E-C\right) \rightarrow \mathcal{O}_{X}\left(H_{p}+E\right) \rightarrow \mathcal{O}_{C}\left((H+E)_{C}\right) \rightarrow 0
$$

one obtains a contradiction as above (note that $h^{0}\left(\mathcal{O}_{X}\left(H_{p}+E\right)\right)=\chi\left(\mathcal{O}_{X}\left(H_{p}+E\right)\right)$ because $H_{p}+E=H_{p}+K_{X}$, and $\left.\chi\left(\mathcal{O}_{X}\left(H_{p}+E\right)\right)=\frac{1}{2}(H+E) \cdot H=1\right)$.

Case 2. Suppose now the general $C \in|L|$ has more than one $g_{d}^{1}$. Then $d=4$ because an hyperelliptic curve has a unique $g_{2}^{1}$, and a trigonal curve of genus $g>4$ has a unique $g_{3}^{1}$. So suppose the general $C \in|L|$ has gonality 4 and has two distinct $g_{4}^{1}$. The two $g_{4}^{1}$ define a morphism

$$
\psi: C \rightarrow Q=\mathbb{P}^{1} \times \mathbb{P}^{1}
$$

of degree $e$ onto a a divisor $B$ of type $(4 / e, 4 / e)$ on $Q$. We cannot have $e=4$, as otherwise $B \cong \mathbb{P}^{1}$ and the two linear series coincide. If $e=2$, then $B$ cannot be rational because $C$ is not hyperelliptic. But $B$ cannot be an elliptic curve because $C$ does not have morphism to curves of genus 1 by Proposition 3.1. Hence $e=1$ and $\psi: C \rightarrow B$ is birational. 
Since $B$ has arithmetic genus 9 , the map $\psi$ is an isomorphism. Thus $B \cong C$ is a smooth curve of type $(4,4)$ on $Q=\mathbb{P}^{1} \times \mathbb{P}^{1}$. We will show that this is not possible by proving that: (a) the curve $C$ has a base point free complete $g_{8}^{2}$ that defines a map $\phi: C \rightarrow \mathbb{P}^{2}$ that is not $4: 1$ onto its image; (b) the only base point free and complete $g_{8}^{2}$ on a divisor $B$ of type $(4,4)$ on $Q$ are $\left|\mathcal{O}_{B}(2,0)\right|$ and $\left|\mathcal{O}_{B}(0,2)\right|$, and these define 4 : 1 maps.

We now show a general $C$ in $|L|$ has a complete and base point free $g_{8}^{2}$ that defines a morphism $g: C \rightarrow \mathbb{P}^{2}$ that is not $4: 1$ onto its image. Recall $X$ is the blow up of the abelian surface $S=\operatorname{Jac}(Y) \cong \operatorname{Pic}(Y)$ at the origin $0_{S} \cong \mathcal{O}_{Y}\left(K_{Y}\right)$. Given a point $p$ in $Y$ we denote by $\theta_{p}$ the theta divisor

$$
\theta_{p}=\left\{\mathcal{O}_{Y}(p+y) \mid y \in Y\right\}
$$

If $p+p^{\prime}=K_{Y}$, the divisor $\theta_{p}+\theta_{p^{\prime}}$ is symmetric with respect to the involution of the abelian surface, and defines a morphism $S \rightarrow \mathbb{P}^{3}$ whose image is a quartic Kummer surface $T$. The pull back of $\theta_{p}+\theta_{p^{\prime}}$ to $X$ is the divisor $H_{p}+H_{p^{\prime}}+2 E$, which therefore defines a $2: 1$ morphism $X \rightarrow \mathbb{P}^{3}$ whose image is the Kummer surface $T$. This morphism maps $C$ birationally onto its image in $T$ because $C$ has no $2: 1$ morphism to a curve, as it is not hyperelliptic and does not have any morphism of degree $d \geq 2$ onto a nonrational curve.

Next we project the Kummer surface from the node that is the image of $0_{S} \cong$ $\mathcal{O}_{Y}\left(K_{Y}\right)$ : this amounts to consider the morphism $X \rightarrow \mathbb{P}^{2}$ defined by $H_{p}+H_{p^{\prime}}$ (one can check $h^{0}\left(X, \mathcal{O}_{X}\left(H_{p}+H_{p^{\prime}}\right)\right)=3$ using Proposition 1.2.ii). This projection is a degree 2 morphism $\gamma: T \rightarrow \mathbb{P}^{2}$. Since $C$ maps birationally onto its image in $T$, the restriction $g: C \rightarrow \mathbb{P}^{2}$ of $\gamma$ to $C$ is either birational or $2: 1$ onto its image, in any case is not $4: 1$.

To finish, observe that $g^{*}\left(\mathcal{O}_{\mathbb{P}^{2}}(1)\right)$ is the line bundle corresponding to the divisor $\left.\left(H_{p}+H_{p^{\prime}}\right)\right|_{C}$. We claim $\left.\left(H_{p}+H_{p^{\prime}}\right)\right|_{C}$ moves in a complete $g_{8}^{2}$ on $C$. To compute $\left.\mathcal{O}_{C}\left(H_{p}+H_{p^{\prime}}\right)\right|_{C}$, we recall $h^{0}\left(X, \mathcal{O}_{X}\left(H_{p}+H_{p^{\prime}}\right)\right)=3$ and look at the exact sequence

$$
0 \rightarrow \mathcal{O}_{X}\left(H_{p}+H_{p^{\prime}}-C\right) \rightarrow \mathcal{O}_{X}\left(H_{p}+H_{p^{\prime}}\right) \rightarrow \mathcal{O}_{C}\left(\left.\left(H_{p}+H_{p^{\prime}}\right)\right|_{C}\right) \rightarrow 0 .
$$

Since $C-H_{p}-H_{p^{\prime}} \equiv H+E$ is big and nef by 1.3 , the $H^{0}$ and $H^{1}$ of $H_{p}+H_{p^{\prime}}-C$ vanish, hence

$$
h^{0}\left(C, \mathcal{O}_{C}\left(\left.\left(H_{p}+H_{p^{\prime}}\right)\right|_{C}\right)\right)=3 .
$$

Summing up we have found a complete $g_{8}^{2}$ on $C$ that defines a morphism $g: C \rightarrow$ $\mathbb{P}^{2}$ that is not $4: 1$ onto its image.

To complete the proof, we need to show that, if $B$ is a smooth divisor of type $(4,4)$ on the quadric surface $Q \subset \mathbb{P}^{3}$, then the only base point free and complete $g_{8}^{2}$ on $B$ are $\left|\mathcal{O}_{B}(2,0)\right|$ and $\left|\mathcal{O}_{B}(0,2)\right|$. So let $\delta$ be an effective divisor of degree 8 on $B$ such that $|\delta|$ is a complete and base point free $g_{8}^{2}$. Then $h^{0}\left(B, \mathcal{O}_{B}(\delta)\right)=3$ and we may assume that $\delta$ consists of 8 distinct points.

By Riemann-Roch

$$
h^{0}\left(B, \mathcal{O}_{B}\left(K_{B}-\delta\right)\right)=3+\operatorname{deg}\left(K_{B}-\delta\right)+1-g(B)=3 .
$$

Since $B$ is a divisor of type $(4,4)$, by adjunction $K_{B}=2 H$, where $\mathcal{O}_{B}(H)=\mathcal{O}_{B}(1,1)$. Therefore $\mathcal{O}_{B}\left(K_{B}-\delta\right)=\mathcal{I}_{\delta, B}(2 H)$, and

$$
h^{0}\left(Q, \mathcal{I}_{\delta, Q}(2,2)\right)=h^{0}\left(B, \mathcal{I}_{\delta, B}(2 H)\right)=3
$$


because $H^{0}\left(Q, \mathcal{I}_{B, Q}\right)=H^{1}\left(Q, \mathcal{I}_{B, Q}\right)=0$. Thus we see that the linear system

$$
\mathcal{D}=\left|\mathcal{O}_{Q}(2,2)-\delta\right|
$$

cut out on $Q$ by quadric surfaces containing $\delta$ has projective dimension 2 .

If the linear system $\mathcal{D}$ had no fixed component, then two general elements in the linear system would meet properly in a zero dimensional scheme of degree 8 containing $\delta$, hence equal to $\delta$. Thus $\delta$ would be a complete intersection of three quadrics in $\mathbb{P}^{3}$, contradicting $h^{0}\left(Q, \mathcal{I}_{\delta, Q}(2,2)\right)=3$. Thus $\mathcal{D}$ has a fixed curve, say $D$. We write $\delta=\alpha+\beta$ where $\alpha$ consists of those points of $\delta$ that are in the support of $D$.

Case a: $D$ is a line, with respect to the embedding of $Q$ in $\mathbb{P}^{3}$ by $\mathcal{O}_{Q}(1,1)$, say of type $(0,1)$.

Then the linear system $\mathcal{D}$ is, up to removing $D$, the linear system

$$
\left|\mathcal{O}_{Q}(2,1)-\beta\right| .
$$

Observe that $\beta$ cannot contain 3 collinear points, otherwise the line through them would be contained in the fixed component of $\mathcal{D}$. Similarly, if $\beta$ had 4 points in a plane $\Pi$, then there would be a fixed component of $\mathcal{D}$ contained in $\Pi$ and different from $D$, which is absurd.

Since $D$ is a line and $\alpha \subset D \cap B$, there are at most four points in $\alpha$, so $\beta$ contains at least 4 points $P_{1}, P_{2} P_{3}$ and $P_{4}$. As $\beta$ has no 3 collinear points, and no 4 coplanar points, the points $P_{i}$ impose independent conditions on $\left|\mathcal{O}_{Q}(2,1)\right|$ : a divisor of type $(2,1)$ containing only the first three points is given by a conic through $P_{1}, P_{2}$, and $P_{3}$, plus a line not containing $P_{4}$. Then $\operatorname{dim} \mathcal{D} \leq \operatorname{dim}\left|\mathcal{O}_{Q}(2,1)\right|-4=1$, a contradiction.

Thus this case does not occur.

Case b: $D$ is a plane section of $Q$, that is a divisor of type $(1,1)$.

Then the linear system $\mathcal{D}$ is, up to removing $D$, the linear system

$$
\left|\mathcal{O}_{Q}(1,1)-\beta\right| .
$$

Since $\mathcal{D}$ has projective dimension 2 , we see that $\beta$ consists of at most one point. If $\beta=P$ had degree one, then $\alpha$ would have degree 7 besides being contained in the plane of $D$. Thus

$$
h^{0}(B, \mathcal{O}(\alpha)) \geq h^{0}\left(B, \mathcal{O}_{B}(1,1)\right)-1=3 .
$$

But $\alpha \leq \delta$ and $h^{0}\left(B, \mathcal{O}_{B}(\delta)\right)=3$ by assumption, so the unique point of $\beta$ is a base point of $\delta$, contradicting the fact that $\delta$ is base point free. Thus $\beta$ is empty. But then $\delta=\alpha$ is a plane section, and this is also a contradiction because $h^{0}\left(B, \mathcal{O}_{B}(1,1)\right)=4$. Thus this case also does not occur.

Case c: $D$ has degree 3 and arithmetic genus 0 , that is, it is a divisor of type $(2,1)$ or $(1,2)$.

Suppose that $D$ has type $(1,2)$. Then the linear system $\mathcal{D}$ is, up to removing $D$, the linear system

$$
\left|\mathcal{O}_{Q}(1,0)-\beta\right|
$$

Since $\left|\mathcal{O}_{Q}(1,0)\right|$ has projective dimension 1 , this case does not occur. 
Case d: $D$ has degree 2 and arithmetic genus -1 , that is, it is a divisor of type $(2,0)$ or $(0,2)$.

Suppose that $D$ has type $(0,2)$. Note that $D$ is either the disjoint union of two lines of type $(0,1)$, or a double structure on a line of type $(0,1)$.

The the linear system $\mathcal{D}$ is, up to removing $D$, the linear system

$$
\left|\mathcal{O}_{Q}(2,0)-\beta\right| \text {. }
$$

Since $\left|\mathcal{O}_{Q}(2,0)\right|$ has projective dimension 2 and no base points, $\beta$ is the zero divisor. Thus $\delta=\alpha$ is contained in the fixed curve $D$. We know that at most four points of $\delta$ are collinear, because $\delta$ lies on the curve $B$ which has type $(4,4)$. Therefore the only possibility is that $D$ is the union of two lines $L_{1}$ and $L_{2}$ of type $(0,1)$, and $\delta=B . D$. Therefore $\mathcal{O}_{B}(\delta) \cong \mathcal{O}_{B}(0,2)$ (or $\mathcal{O}_{B}(\delta) \cong \mathcal{O}_{B}(2,0)$ if $D$ has type $(2,0)$ ).

There are no other possibilities for $D$, because it is contained properly in a divisor of type $(2,2)$. Thus we have proven that the only complete and base point free $g_{8}^{2}$ 's on $B$ are $\mathcal{O}_{B}(2,0)$ and $\mathcal{O}_{B}(0,2)$.

4. Number of moduli of genus 9 primitive and solvable coverings of the projective line. In this section we show that, if $C$ is a general curve in a 10 dimensional family of smooth curves of genus 9 , then a finite map $f: C \longrightarrow \mathbb{P}^{1}$ that has a primitive and solvable Galois group has degree at most 4 (the Galois group is primitive when $f$ cannot be factored nontrivially). The proof is based on a counting argument due to Zariski [21].

Given a finite morphism $f: C \longrightarrow C_{0}$ of smooth curves, we denote by $b(q)$ the multiplicity of a branch point $q$ of $f$ in the branch divisor.

Theorem 4.1 (Zariski, see [18, Proposition 3.1]). Let $f: C \longrightarrow C_{0}$ be a degree $d$ primitive solvable covering of curves. Then there exists a prime $p$ such that $d=p^{k}$, and for every branch point $q$ of $f$ the multiplicity $b(q)$ is bounded by the formula:

$$
b(q) \geq \frac{p^{k}-p^{k-1}}{2} .
$$

Moreover, if $p=2$ and $d-1$ is prime, then $b(q) \geq 2^{k-1}-1$.

We recall Zariski's argument. Let $S_{d}=\operatorname{Aut}(\Omega)$, where $\Omega$ is a set of $d$ elements. Fix $x \in \Omega$ and consider a primitive solvable subgroup $G$ of $S_{d}$. It is well known that $G$ has only one minimal normal subgroup $A$, which is an elementary abelian $p$-group for some prime $p$. Moreover, $G$ is the semidirect product $G=A \rtimes G_{x}$, where $G_{x}$ denotes the stabilizer of $x$ in $G$. Since the action of $A$ on $\Omega$ is regular, the cardinality of $A$ is equal to $d=p^{k}$ for some $k \geq 1$. Furthermore, identifying $\Omega$ with the vector space $A$, the group $G$ acts as a subgroup of the group $\operatorname{Aff}(A)$ of affinities of $A$. Hence an element $g \in G$, not equal to the identity $1_{G}$, has at most $p^{k-1}$ fixed points. To each branch point $q$ the monodromy representation associates an element $g \in G$ whose action on $\Omega$ has $d-b(q)$ orbits. Hence $b(q) \geq \frac{d-n}{2}$ where $n$ is the number of fixed points of $g$.

Proposition 4.2. Let $\mathcal{C}$ be an irreducible family of curves of genus 9 whose general curve is a degree $d$ primitive and solvable covering of $\mathbb{P}^{1}$. Then the dimension of $\mathcal{C}$ is at most 9 unless $d \leq 5$.

Proof. Consider a family $\mathcal{C}$ of curves $C$ of genus 9 such that the general curve $C$ admits a degree $d$ primitive solvable covering of $\mathbb{P}^{1}$ with $r$ distinct branch points, each with multiplicity at least $m$. 
By the Riemann Hurwitz formula the degree of the branch divisor $B$ of the covering $f: C \rightarrow \mathbb{P}^{1}$ is $16+2 d$. If $f$ has exactly $r$ distinct branch points, each with multiplicity at least $m$, then $\operatorname{deg}(B) \geq r m$, therefore

$$
r \leq \frac{16+2 d}{m}
$$

The dimension of $\mathcal{C}$ is then at most

$$
r-3 \leq \frac{16+2 d}{m}-3
$$

By (4.1) $d=p^{k}$ and

$$
m \geq \frac{p^{k}-p^{k-1}}{2}
$$

Thus

$$
\operatorname{dim} \mathcal{C} \leq 4 \frac{8+p^{k}}{p^{k}-p^{k-1}}-3=\frac{32}{p^{k-1}(p-1)}+\frac{4}{1-1 / p}-3
$$

This shows $\operatorname{dim} \mathcal{C} \leq 9$ unless $d \leq 5$ or $d=8$. But when $d=8$ we can use the better estimate $m \geq 2^{k-1}-1=3$ to conclude $\operatorname{dim} \mathcal{C}<8$.

Proposition 4.3. If $C$ is a general curve in a 10 dimensional family of smooth genus 9 curves, then there is no degree 5 covering $C \rightarrow \mathbb{P}^{1}$ with a primitive solvable Galois group.

Proof. Let $f: C \rightarrow \mathbb{P}^{1}$ be a degree 5 primitive and solvable covering. By Riemann-Hurwitz the branch divisor $B_{f}$ has degree 26. By (4.1) every branch point $q$ has multiplicity $b(q) \geq 2$, hence the number $r$ of distinct branch points has to be $\leq 13$.

The Hurwitz scheme of coverings of $\mathbb{P}^{1}$ having $r$ distinct branch points has dimension $r-3$. Thus, if $C$ varies in a family of dimension $\geq 10$, the only possibility is that $r=13$ and $b(q)=2$ for every branch point $q$. Let $G \subset S_{5}$ be the Galois group of $f$. In the Galois group, for any branch point $q_{i} \in B_{f}$, there is an associated cycle $g_{i} \in G$ with $3=d-b\left(q_{i}\right)$ orbits. Furthermore by Zariski's argument the cycle $g_{i}$ has at most one fixed point, so it must be (12)(34) up to conjugation. Moreover, the product of the cycles $g_{i}$ is the identity of the Galois group.

$$
\prod_{i=1}^{13} g_{i}=1_{G}
$$

As explained after Theorem 4.1, the group $G$ is contained in the group of affinities of $\mathbb{Z}_{5}$, which is a semidirect product $\mathbb{Z}_{5} \rtimes \mathbb{Z}_{4}$. Therefore there is an induced map $\phi: G \rightarrow \mathbb{Z}_{4}$. Since $g_{i}$ has order 2 , it can't be contained in the kernel of $\phi$, so $\phi\left(g_{i}\right)$ is the unique element $h=[2]$ of order 2 in $\mathbb{Z}_{4}$. Then $\phi\left(\prod_{i=1}^{13} g_{i}\right)$ is also equal to $h$, contradicting (1). 
5. Proof of the main result. In this section we collect all the previous results to show that the general curve of the family constructed in section 2 is algebraically, but non rationally, uniformized by radicals.

THEOREM 5.1. Let $\mathcal{C}$ be the family of smooth genus 9 curves $C$ for which there exists a genus 2 curve $Y$ such that $C \subset X=\operatorname{Sym}^{2}(Y)$ and the class of $C$ in the Néron-Severi group of $X$ is $3 H+E$. Then a general curve in $\mathcal{C}$ is algebraically, but non rationally, uniformized by radicals, and provides a counterexample to Statement $S(4,2,9)$ of $[1]$.

Proof. To see that a curve $C$ in the family is algebraically uniformized by radicals is easy. Since $C \subset X=\operatorname{Sym}^{2}(Y)$ and $C \equiv 3 H+E$, for every point $p \in Y$ the curve $C$ intersects the divisor $H_{p} \subset X$ in a scheme of length 4. We define a map $\phi: Y \longrightarrow \operatorname{Sym}^{4}(C)$ sending a point $p \in Y$ to $H_{p} \cdot C$. Then as in [18, Proposition 5.1] we deduce there is a smooth curve $C^{\prime}$ that covers $C$ and admits a morphism $C^{\prime} \rightarrow Y$ of degree $\leq 4$.

We now have to show that a general $C$ in our family is not rationally uniformized by radicals, that is, there does not exist a finite map $C \longrightarrow \mathbb{P}^{1}$ with solvable Galois group. In particular, $C$, contrary to its covering $C^{\prime}$, does not admit a nonconstant map of degree 4 or less to a curve of genus 2 or less, and thus provides a counterexample to $S(4,2,9)$.

Suppose by way of contradiction there is $C \longrightarrow \mathbb{P}^{1}$ with solvable Galois group. Then we can factor it as

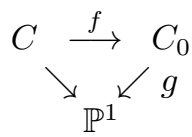

where $f$ is a covering of degree $d \geq 2$ with a primitive and solvable Galois group (the Galois group is primitive when $f$ cannot be factored). Since $C$ does not cover any non rational curve by Proposition 3.1, the curve $C_{0}$ is rational, and we are reduced to show there does not exist a finite map $C \longrightarrow \mathbb{P}^{1}$ with primitive and solvable Galois group. By the dimension count of Propositions 4.2 and 4.3 any such map would have degree $d \leq 4$. But by Theorem 3.2 there are no morphisms $C \rightarrow \mathbb{P}^{1}$ of degree $d \leq 4$. Thus the proof is complete.

\section{REFERENCES}

[1] D. Abramovich And J. Harris, Abelian varieties and curves in $W_{d}(C)$, Compositio Math., 78 (1991), pp. 227-238.

[2] E. Arbarello, M. Cornalba, P. Griffiths, and J. Harris, Geometry of Algebraic Curves, Springer-Verlag, (1985).

[3] A. Beauville, Variétés Kähleriennes dont la première classe de Chern est nulle, J. Differential Geom., 18 (1983), pp. 755-782.

[4] C. Ciliberto AND G. VAN DER Geer, On the Jacobian of a hyperplane section of a surface, in "Classification of irregular varieties (Trento, 1990)", Lecture Notes in Math., 1515, Springer Berlin (1992), pp. 33-40.

[5] A. J. DE JONG AND J. STARR, Every rationally connected variety over the function field of a curve has a rational point, Amer. J. Math., 125 (2003), pp. 567-580

[6] O. Debarre and R. Fahlaoui, Abelian varieties in $W_{r}^{d}(C)$ and points of bounded degree on algebraic curves, Compositio Math., 88 (1993), pp. 235-249.

[7] J. Fogarty, Algebraic families on an algebraic surface, Amer. J. Math., 90 (1968), pp. 511-521.

[8] M. Fried, Combinatorial computation of moduli dimension of Nielsen classes of covers, Contemp. Math., 89 (1989), pp. 61-79, Amer. Math. Soc., Providence, RI. 
[9] R. Friedman, Algebraic surfaces and holomorphic vector bundles, Universitext, SpringerVerlag New York, (1998).

[10] T. Graber, J. Harris, And J. Starr, Families of rationally connected varieties, J. Amer. Math. Soc., 16 (2003), pp. 57-67.

[11] R. Guralnick and M. Neubauer, Monodromy groups of branched coverings: the generic case, in "Recent developments of the inverse Galois problem (Seattle, Wa, 1993)", Contemp. Math., 186 (1995), pp. 325-352, Amer. Math. Soc., Providence, RI.

[12] R. Guralnick AND J. SHAREShian, Symmetric and alternating groups as monodromy groups of Riemann surfaces. I. Generic covers and covers with many branch points, with an appendix by Guralnick and R. Stafford, Mem. Amer. Math. Soc., 189 (2007), vi+128 pp.

[13] J. HARRIS, Galois groups of enumerative problems, Duke Math. J., 46 (1979), pp. 685-724.

[14] S. Kolzumi, The ring of algebraic correspondences on a generic curve of genus g, Nagoya Math. J., 60 (1976), pp. 173-180.

[15] R. Lazarsfeld, Brill-Noether-Petri without Degenerations, J. Diff. Geom., 23 (1986), pp. 299 307.

[16] M. Neubauer, On monodromy groups of fixed genus, J. Algebra, 153 (1992), pp. 215-261.

[17] G. Pirola and E. Schlesinger, Monodromy of projective curves, J. Algebraic Geom, 14 (2005), pp. 623-642.

[18] G. Pirola and E. Schlesinger, A curve algebraically but not rationally uniformized by radicals, J. Algebra, 289 (2005), pp. 412-420.

[19] I. ReIDER, Vector bundles of rank 2 and linear systems on algebraic surfaces, Ann. of Math. (2), 127 (1988), pp. 309-316.

[20] A. N. Tyurin, Cycles, curves and vector bundles on an algebraic surface, Duke Math. J., 54 (1987), pp. 1-26.

[21] O. ZARISKI, Sull'impossibilità di risolvere parametricamente per radicali un'equazione algebrica $f(x, y)=0$ di genere $p>6$ a moduli generali, Atti Accad. Naz. Lincei Rend., Cl. Sc. Fis. Mat. Natur., serie VI, 3 (1926), pp. 660-666. 
G. P. PIROLA, C. RIZZI AND E. SCHLESINGER 